University of Nebraska - Lincoln

DigitalCommons@University of Nebraska - Lincoln

Faculty Publications from the Department of Engineering Mechanics

$9-2005$

\title{
Antiplane surface acoustic waves propagating in elastic half- space coated with an anisotropic laminate
}

\author{
Xiangfa Wu \\ Department of Engineering Mechanics, University of Nebraska-Lincoln, xfwu@unlserve.unl.edu
}

Yuris A. Dzenis

Department of Engineering Mechanics, University of Nebraska-Lincoln, ydzenis@unl.edu

Follow this and additional works at: https://digitalcommons.unl.edu/engineeringmechanicsfacpub

Part of the Mechanical Engineering Commons

Wu, Xiangfa and Dzenis, Yuris A., "Antiplane surface acoustic waves propagating in elastic half-space coated with an anisotropic laminate" (2005). Faculty Publications from the Department of Engineering Mechanics. 25.

https://digitalcommons.unl.edu/engineeringmechanicsfacpub/25

This Article is brought to you for free and open access by the Mechanical \& Materials Engineering, Department of at DigitalCommons@University of Nebraska - Lincoln. It has been accepted for inclusion in Faculty Publications from the Department of Engineering Mechanics by an authorized administrator of DigitalCommons@University of Nebraska - Lincoln. 
Published in Composites Science and Technology 65:11-12 (September 2005), pp. 1761-1768; doi 10.1016/j.compscitech.2005.03.003

Copyright (C) 2005 Elsevier Ltd. Used by permission. http://www.elsevier.com/locate/compscitech

Submitted March 3, 2005; accepted March 10, 2005; published online April 19, 2005.

\title{
Antiplane surface acoustic waves propagating in elastic half-space coated with an anisotropic laminate
}

\author{
Xiang-Fa Wu and Yuris A. Dzenis \\ Department of Engineering Mechanics, Center for Materials Research and Analysis, \\ University of Nebraska-Lincoln, Lincoln, NE 68588-0526, USA \\ Corresponding author - X.-F. Wu, email xfwu@unlserve.unl ; fax 402 472-8292
}

\begin{abstract}
Dispersion relation of antiplane surface acoustic waves (SAWs) propagating in elastic half-space coated with an anisotropic laminate was determined explicitly by means of Stroh's formalism within the subsonic range, where there is no energy leakage into the substrate. During the procedure, the governing dynamic equation in each anisotropic layer and the displacement-traction continuity at interfaces were exactly satisfied by Stroh's functions. Explicit algebraic equation was derived for determining the dispersion relation. As an example, the wave number vs. phase velocity diagram for steel half-space coated with a graphite-fiber/ epoxy laminate with a $\left[ \pm 45^{\circ} / 0_{2}^{\circ}\right.$ ] lay-up was demonstrated. The given method can be used for the study of SAW properties of anisotropic coating systems and non-destructive evaluation based on surface/guided wave methods.
\end{abstract}

Keywords: anisotropic elasticity, Stroh's formalism, surface acoustic waves (SAWs), dispersion relation, composite laminate, anisotropic coatings

\section{Introduction}

With increasing use of surface coatings techniques in aeronautical engineering and semiconductor industry, measurements of near-surface mechanical properties of solids coated with thin materials play an important role in understanding the behaviors of these materials and structures in applications. Among various surfacemeasurement methods, surface/guided wave method is used most extensively, such as the surface Brillouin scattering (SBS), laser-generated surface acoustic waves (LSAWs), acoustic microscopy (AM), and surface acoustic wave (SAW) spectrometry and spectroscopy, etc. [1-5], in which the dispersion relations of SAWs propagating in near-surface layers are employed as theoretical bases for extracting the mechanical properties of the near-surface materials from experimental data. The simplest SAW in elastically isotropic halfspace coated with another elastically isotropic layer is the classic Love wave, which has been well addressed in the literature [6-8]. So far, anisotropic and heterogeneous coating systems such as multilayered thermal barrier coatings and functionally gradient coatings have been commonly used in enhancing the thermal stability and the resistance to chemical corrosion and mechanical wearing of metallic substrates. In an attempt to evaluate the mechanical properties of these surface coatings by means of surface/guided wave methods, it is desirable to study their SAW dispersion relations. In the past four decades, a number of investigators have contributed significantly in this field $[9,10]$. The principal aspect of the wave spectra of an elastic substrate coated with a single layer has been well explored in the literature, which consists of the supersonic leaking waves and subsonic localized SAWs [11]. Both of them are dispersive, while the latter does not have energy leakage into the substrate. Therefore, subsonic SAWs may be used for the characterization of the surface layers. Re- 
cently, wave dispersion relations of anisotropic halfspace coated with another anisotropic surface layer has been investigated in details by Shuvalov and Every [11], who obtained a family of subsonic SAW dispersion curves relevant to various frequency ranges and materials. In their work, the impedance method was employed, which was initially used in understanding surface and interface waves in anisotropic materials [12-14]. SAW dispersion relations of anisotropic multilayers are of particular importance to modern surface coatings science and technologies, however, not much work has been done yet in this filed. Unlike homogenous elastic medium coated with a single layer, the dynamic governing equations of SAWs in multilayered surface coatings involve multiple displacement-traction continuities at interfaces, which generally lead to a coupled system of wave equations. Physically, wave propagation in laminates invokes complicated wave reflection, refraction, and deflection at ply interfaces and laminate surfaces. This makes experimental evaluation much more arduous, especially in the high frequency range where the size of microdamages and ply thickness are comparable to the wavelengths.

For wave propagation in isotropic multilayers, quite a few methods have been contributed to characterize its wave dispersion in the past decades, and exact wave dispersion relations have been documented in wellknown monographs [7-8, 15-16]. For anisotropic laminates, historically, Farnell and Adler [17-18] first proposed the partial wave method. Murakami [19] and Murakami and Akiyama [20] developed a mixture theory to approach the wave dispersion relations in angleply laminates. In their model the elemental cell with ply thickness was introduced to approximate the elastic field across the ply thickness, and interface continuity conditions were satisfied in the weak form of Reissner's variation. Utilizing eigenvector expansions of the dynamic governing equations of each ply of anisotropic laminates, Liu et al. [21] obtained the exact dispersion relations for Lamb wave propagating in the laminates. Meanwhile, Liu et al. [22] further developed a purely numerical method based on finite strip elements to explore the wave dispersions in composite laminates. Applications of Liu's method in various wave phenomena regarding anisotropic laminates may be found in the monograph authored by Liu and Xi [23]. A more efficient approach for multilayers, typically called the transfer matrix or matrix method, was initially proposed by Adler et al. [24] and Adler [25]. The matrix method is known to have computational instabilities in some cases. Improvements of this method have been made by Smith [26] and Tan [27] using Green's functions. Moreover, Sun et al. [28] developed an efficient method based on a reduced-laminate model to capture the wave dispersion relations in thick composite laminates made of angle-plies. In their model, the effective moduli for thick-section composite laminates were used to represent the laminates as homogeneous but anisotropic media. Their results indicated that for long wavelengths the effective modulus solutions converge to the exact ones, while with the decrease of wavelength, the effective modulus solutions start to deviate from the exact ones, depending on the mode of wave motion, stacking sequence, and the number of plies in the laminate. Recently, Caviglia and Morro [29-30] further considered the mathematical formulations of wave propagation in anisotropic multilayers using a Riccati-type evolution equation to accommodate the interface continuities. The wave reflection and transmission ratios across the interfaces were demonstrated in their model. As a fact, fundamentals of wave propagation in layered anisotropic media may be found in the recent monograph by Neyfeh [31].

One may find that the above works for anisotropic laminates were largely based on the general solutions to elastodynamic equations of anisotropic media without considering the solution structures. Therefore, the resulted dispersion relations in these models are generally implicit. As a matter of fact, Stroh's formalism [14, 3233] has been proved as an efficient and concise method for describing the elastic behaviors for anisotropic elastic materials, which is a more generalized formalism compared with the classic Lekhnitskii's formalism [34]. In his monograph, Ting [14] summarized the generalized Stroh's formalism for the study of steady bulk, surface, and interface wave phenomena in anisotropic media. In this work, we further utilize Stroh's formalism to study a simple steady phenomenon of antiplane SAWs propagating in multilayered anisotropic surface coatings. Based on this formalism, the governing dynamic equation of each anisotropic ply and the substrate, interface continuities, and coating surface boundary conditions can be satisfied exactly by the Stroh's functions. During the procedure, by means of Stroh's formalism $[14,32-33]$, the general solution to each anisotropic layer can be expressed directly in terms of analytic functions. One of the advantages of this method is that the obtained SAW dispersion relation is in closed-form, and the corresponding numerical procedure is shown to be very robust and independent of the layer number. This method can be furthered parallelly for the study of dispersion relations of steady inplanar SAWs propagating in multilayered anisotropic surface coatings and other layered material systems with damages. This method may provide a solid basis for laminate non-destructive evaluation (NDE), etc.

\section{Antiplane elasticity of anisotropic solids}

In the absence of body forces, the elastodynamic equations for solid materials are 


$$
\sigma_{i j, j}=\rho \frac{\partial^{2} u_{i}}{\partial t^{2}}
$$

where $u_{i}(i=1,2,3)$ is the displacement of the material point, $\rho$ is the mass density, the repeated indices imply the summation, and a comma stands for differentiation. The generalized Hooke's law for a homogenous, anisotropic, and linearly elastic solid is given by

$$
\sigma_{i j}=C_{i j k l} \varepsilon_{k l},
$$

where $\varepsilon_{i j}=1 / 2\left(u_{i, j}+u_{j, i}\right)$ denotes the linearly infinitesimal strain tensor, and $C_{i j k l}$ is the elastic stiffness tensor satisfying the usual symmetry. Due to the symmetry of $C_{i j k l}$ relation (2) can be rewritten as

$$
\sigma_{i j}=C_{i j k l} u_{k, l} \text {. }
$$

For a general anisotropic elastic material, the antiplane and inplane deformations are coupled, thus no pure antiplane or inplane deformation exists. For some special anisotropic materials with the elastic stiffness $C_{i j k l}$ in terms of a contracted matrix such that

$$
\mathbf{C}=\left[\begin{array}{llllll}
C_{11} & C_{12} & C_{13} & 0 & 0 & C_{16} \\
& C_{22} & C_{23} & 0 & 0 & C_{26} \\
& & C_{33} & C_{34} & C_{35} & C_{36} \\
& & & C_{44} & C_{45} & C_{46} \\
& \text { sym. } & & & C_{55} & C_{56} \\
& & & & & C_{66}
\end{array}\right],
$$

the antiplane and inplane deformations can be decoupled, and such anisotropic materials are capable of an pure antiplane deformation [14], for example the general monoclinic materials with $C_{34}$ and $C_{35}$ equal to zero and with a plane of symmetry at $x_{3}=0$.

In the case of two-dimensional elastodynamic problems, the Cartesian coordinate system is chosen such that the antiplane deformation is in the $x_{3}$-direction. Let $u, v$, and $w$ represent the displacement components in the $x_{1}$, $x_{2}$ and $x_{3}$-directions, respectively. For a steady $\mathrm{SH}$-wave propagating along $x_{1}$-axis, only the antiplane deformation is evoked such that:

$$
u=v=0, \quad w=w\left(x_{1}-c t, x_{2}\right) .
$$

There the relevant nontrivial shear stresses are $\tau_{31}$ and $\tau_{32}$, and $c$ is the wave phase velocity. If the material stiffness matrix is in form of (4), the equilibrium equations in the $x_{1}$ and $x_{2}$-directions are satisfied naturally, and the equilibrium equation in the $x_{3}$-direction leads to

$$
\left(C_{55}-\rho c^{2}\right) \frac{\partial^{2} w}{\partial x_{1}^{2}}+2 C_{45} \frac{\partial^{2} w}{\partial x_{1} \partial x_{2}}+C_{44} \frac{\partial^{2} w}{\partial x_{2}^{2}}=0,
$$

where the differential relation $\partial^{2} / \partial t^{2}=c^{2} \partial^{2} / \partial x_{1}^{2}$ has been introduced. Equation (6) is the governing equation for an anisotropic body under antiplane deformation. The nontrivial stresses $\tau_{13}, \tau_{23}$, and $\sigma_{33}$ may be expressed in terms of $w\left(x_{1}, x_{2}\right)$ as follows:

$$
\begin{aligned}
& \tau_{13}=C_{55} \frac{\partial w}{\partial x_{1}}+C_{45} \frac{\partial w}{\partial x_{2}}, \quad \tau_{23}=C_{45} \frac{\partial w}{\partial x_{1}}+C_{44} \frac{\partial w}{\partial x_{2}}, \\
& \sigma_{33}=C_{35} \frac{\partial w}{\partial x_{1}}+C_{34} \frac{\partial w}{\partial x_{2}} .
\end{aligned}
$$

For monoclinic materials with the symmetric plane of $x_{3}=0, \sigma_{33}=0$.

\section{Formal solution of an anisotropic laminate under antiplane deformation}

Consider steady antiplane SAWs propagating in elastic half-space coated with an anisotropic laminate made of $n$ elastically anisotropic piles as shown in Figure 1. The boundary conditions of displacement-traction continuity at interfaces, surface traction-free, and displacement and traction bound at infinity can be expressed as follows:

(1) Interface displacement-traction continuities $\left(x_{2}=h_{i}\right)$

$$
\begin{aligned}
& w\left(x_{1}, h_{i}^{+}\right)=w\left(x_{1}, h_{i}^{-}\right), \quad \tau_{23}\left(x_{1}, h_{i}^{+}\right)=\tau_{23}\left(x_{1}, h_{i}^{-}\right), \\
& (-\infty<x<+\infty, i=2,3, \ldots, n-1) .
\end{aligned}
$$

(2) Traction-free surface condition

$$
\tau_{23}\left(x_{1}, h_{1}\right)=0, \quad(-\infty<x<+\infty) .
$$

(3) Displacement and traction bound at infinity $\left(x_{2} \rightarrow-\infty\right)$

$$
w_{3}\left(x_{1},-\infty\right)=0, \quad \tau_{23}\left(x_{1},-\infty\right)=0, \quad(-\infty<x<+\infty) .
$$

Now let us consider the formal solution of the anisotropic material system shown in Figure 1. By means of the generalized Stroh's formalism for steady waves propagating in anisotropic materials [14], the displacement field may be expressed as

$$
w\left(x_{1}-c t, x_{2}\right)=\frac{-\mathrm{i}}{2 \mu} f(z), \quad z=x_{1}-c t+p x_{2} .
$$

In relation (11), $f$ is an arbitrary purely imaginary function with respect to its argument $z, \mathrm{i}=\sqrt{-1}$, and $p$ is a constant determined by the following characteristic equation:

$$
\left(C_{55}-\rho c^{2}\right)+2 C_{45} p+C_{44} p^{2}=0 .
$$

Equation (12) yields two roots for $p$, one of which is

$$
p=\left(-C_{45}+\mathrm{i} \mu\right) / C_{44}, \quad \mu=\sqrt{C_{44}\left(C_{55}-\rho c^{2}\right)-C_{45}^{2}}
$$

and the other is its complex conjugate $\bar{P}$. When $c=0, \mu=$ $\left(C_{44} C_{55}-C_{45}^{2}\right)^{1 / 2}$ is the principal minor of the stiffness matrix $C_{i j}$ which is always positive for realistic anisotropic 


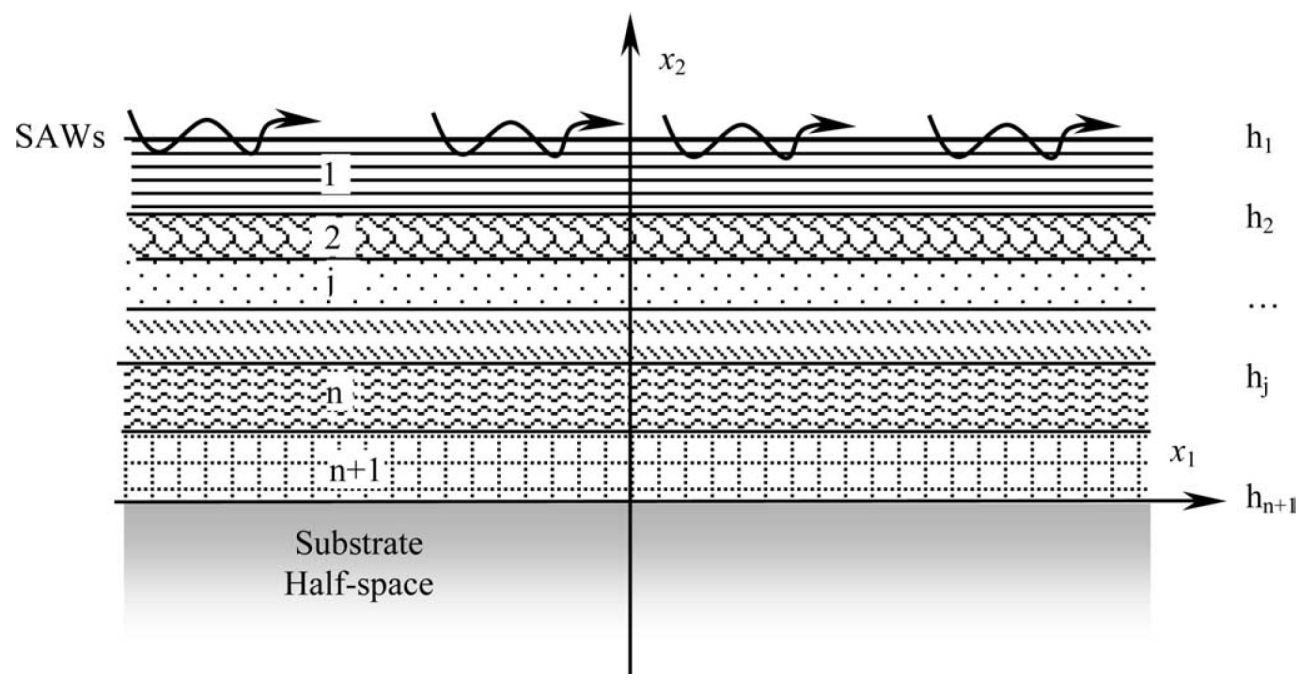

Figure 1. SAWs propagating in elastic half-space coated with an anisotropic laminate.

materials. The stress components $\tau_{13}$ and $\tau_{23}$ can be expressed as

$$
\begin{aligned}
\tau_{13} & =1 / \mu \operatorname{Im}\left[\left(C_{55}+p C_{45}\right) f^{\prime}(z)\right]=-\operatorname{Re}\left[p f^{\prime}(z)\right], \\
\tau_{23} & =1 / \mu \operatorname{Im}\left[\left(C_{45}+p C_{44}\right) f^{\prime}(z)\right]=\operatorname{Re}\left[f^{\prime}(z)\right],
\end{aligned}
$$

where the prime indicates the derivative with respect to $z, \operatorname{Im}($ ) and $\operatorname{Re}($ ) stand for the imaginary and real parts of a complex function, respectively. In a more generalized sense, the above expressions can be further simplified by adopting a stress potential $\phi$ proposed by $\mathrm{Wu}[35]$ such that

$$
\tau_{13}=-\phi, 2, \quad \tau_{23}=\phi,,_{1},
$$

where

$$
\phi=\operatorname{Re}[f(z)] .
$$

Thus, the formal antiplane solution for $w\left(x_{1}, x_{2}\right)$ and $\phi$ can be expressed as

$$
w=\frac{1}{2 \mathrm{i} \mu}[f(z)-\bar{f}(\bar{z})], \quad \phi=1 / 2[f(z)+\bar{f}(\bar{z})],
$$

or

$$
\phi+\mathrm{i} \mu w=f(z) .
$$

For harmonic SAWs propagating along $x_{1}$-axis as shown in Figure 1, the unknown function $f(z)$ in (11) may be written in the following form:

$$
f\left(x_{1}-c t, x_{2}\right)=g\left(x_{2}\right) \exp \left[\mathrm{i} k\left(x_{1}-c t\right)\right],
$$

where $g\left(x_{2}\right)$ is a complex function with respect to $x_{2}$ and $k$ is the wave number. It is known from (11) that the function $f\left(x_{1}-c t, x_{2}\right)$ in (19) is analytic with respect to complex variable $\left(x_{1}-c t+p x_{2}\right)$, thus $g\left(x_{2}\right)$ in the laminate plies and the substrate must have the form:

$$
g\left(x_{2}\right)=\left\{\begin{array}{l}
G_{1} \exp \left(\mathrm{i} k p x_{2}\right)+G_{2} \exp \left(\mathrm{i} k \bar{p} x_{2}\right), \quad\left(x_{2}>0\right), \\
G_{1} \exp \left(\mathrm{i} k \bar{p} x_{2}\right), \quad\left(x_{2}<0\right),
\end{array}\right.
$$

where $G_{1}$ and $G_{2}$ are two complex numbers to be determined according to the boundary conditions, and the choice of $g\left(x_{2}\right)$ at $x_{2}<0$ satisfies the possible existence of subsonic wave without energy leakage into the substrate. In relation (20) the decay property of the SAWs in the substrate has been considered due to $p$ always with the positive imaginary part as assumed in Stroh's formalism. As a result, relation (19) can be rewritten as

$$
\begin{aligned}
& f\left(x_{1}-c t, x_{2}\right) \\
& \quad=\left\{\begin{array}{c}
{\left[G_{1} \exp \left(\mathrm{i} k p x_{2}\right)+G_{2} \exp \left(\mathrm{i} k \bar{p} x_{2}\right)\right] \exp \left[\mathrm{i} k\left(x_{1}-c t\right)\right],} \\
\quad\left(x_{2}>0\right), \\
G_{1} \exp \left[\mathrm{i} k\left(x_{1}-c t+\bar{p} x_{2}\right)\right], \quad\left(x_{2}<0\right) .
\end{array}\right.
\end{aligned}
$$

Here $f$ is a purely imaginary function since (21) is the complete solution involving the conjugate of $p$ based on the formal solution (11). With the aid of Figures (11) and (21), the displacement and traction along an arbitrary line (plane) parallel to $x_{1}$-axis can be expressed as

$$
\begin{aligned}
& 2 \mathrm{i} w\left(x_{1}-c t, x_{2}\right) \\
& =\left\{\begin{array}{c}
1 / \mu\left[G_{1} \exp \left(\mathrm{i} k p x_{2}\right)+G_{2} \exp \left(\mathrm{i} k \bar{p} x_{2}\right)\right] \exp \left[\mathrm{i} k\left(x_{1}-c t\right)\right], \\
\left(x_{2}>0\right), \\
1 / \mu\left\{G_{1} \exp \left[\mathrm{i} k\left(x_{1}-c t+\bar{p} x_{2}\right)\right]\right\}, \quad\left(x_{2}<0\right),
\end{array}\right. \\
& \frac{2 \tau_{23}\left(x_{1}-c t, x_{2}\right)}{\mathrm{i} k} \\
& =\left\{\begin{array}{c}
{\left[G_{1} \exp \left(\mathrm{i} k p x_{2}\right)-G_{2} \exp \left(\mathrm{i} k \bar{p} x_{2}\right)\right] \exp \left[\mathrm{i} k\left(x_{1}-c t\right)\right],} \\
\left(x_{2}>0\right), \\
G_{1} \exp \left[\mathrm{i} k\left(x_{1}-c t+\bar{p} x_{2}\right)\right], \quad\left(x_{2}<0\right) .
\end{array}\right.
\end{aligned}
$$

Equations (22) and (23) can be reformulated in matrix form: 


$$
\begin{aligned}
& \left\{\begin{array}{l}
2 \mathrm{i} w\left(x_{1}-c t, x_{2}\right) \\
\frac{2 \tau_{23}\left(x_{1}-c t, x_{2}\right)}{\mathrm{i} k}
\end{array}\right\} \\
& =\left\{\begin{array}{l}
\mathbf{H} \mathbf{\Lambda}\left(k, x_{2}\right)\left\{\begin{array}{l}
G_{1} \\
G_{2}
\end{array}\right\} \exp \left[\mathrm{i} k\left(x_{1}-c t\right)\right], \quad\left(x_{2}>0\right), \\
{\left[\begin{array}{l}
1 / \mu \\
1
\end{array}\right] G_{1} \exp \left(\mathrm{i} k \bar{p} x_{2}\right) \exp \left[\mathrm{i} k\left(x_{1}-c t\right)\right], \quad\left(x_{2}<0\right),}
\end{array}\right.
\end{aligned}
$$

where $\mathbf{H}$ and $\boldsymbol{\Lambda}\left(k, x_{2}\right)$ are two nonsingular complex $2 \times 2$ matrices defined by

$$
\mathbf{H}=\left[\begin{array}{ll}
1 / \mu & 1 / \mu \\
1 & -1
\end{array}\right], \quad \boldsymbol{\Lambda}\left(k, x_{2}\right)=\left[\begin{array}{ll}
\exp \left(\mathrm{i} k p x_{2}\right) & 0 \\
0 & \exp \left(\mathrm{i} k \bar{p} x_{2}\right)
\end{array}\right] .
$$

Now let us consider the interface continuity of an anisotropic coating system. In an attempt to further simplify the derivation, here we express the elastic field (24) in the $i$ th ply as

$$
v\left(x_{1}-c t, x_{2}\right)^{(i)}=\left\{\begin{array}{l}
2 \mathrm{i} w\left(x_{1}-c t, x_{2}\right) \\
\frac{2 \tau_{23}\left(x_{1}-c t, x_{2}\right)}{\mathrm{i} k}
\end{array}\right\}^{(i)},
$$

where superscript $i$ indicates the $i$ th ply of the laminate as shown in Figure 1. With the help of (26), the displacement-traction continuity along the $i$ th interface can be expressed as

$$
\begin{array}{r}
\tilde{v}\left(x_{1}-c t, h_{i}\right)^{(i-1)}=\tilde{v}\left(x_{1}-c t, h_{i}\right)^{(i)}, \\
(-\infty<x<+\infty, i=2,3, \ldots, n) .
\end{array}
$$

The surface boundary conditions can be represented in the similar way.

\section{SAW dispersion relations}

By repeatedly using relation (27) and the properties of matrices $\mathbf{H}$ and $\boldsymbol{\Lambda}\left(x_{2}, h_{i}\right)$ in (25), the interface continuity of the surface laminate can be finally reduced into a single matrix equation relating the surface boundary conditions and displacement-traction conditions at the interface between the laminate and the substrate such that

$$
\begin{aligned}
& \left\{\begin{array}{l}
2 \mathrm{i} w\left(x_{1}-c t, h_{1}\right) \\
\frac{2 \tau_{23}\left(x_{1}-c t, h_{1}\right)}{\mathrm{i} k}
\end{array}\right\}^{(1)}=\mathbf{B}\left\{\begin{array}{l}
2 \mathrm{i} w\left(x_{1}-c t, h_{n+1}\right) \\
\frac{2 \tau_{23}\left(x_{1}-c t, h_{n+1}\right)}{\mathrm{i} k}
\end{array}\right\}^{(n)}, \\
& (-\infty<x<+\infty),
\end{aligned}
$$

where

$$
\begin{aligned}
& \mathbf{B}=\left[\begin{array}{ll}
B_{11}(k, p, \Delta h) & B_{12}(k, p, \Delta h) \\
B_{21}(k, p, \Delta h) & B_{22}(k, p, \Delta h)
\end{array}\right]=\Pi_{i=1}^{n} \mathbf{S}_{i}, \\
& \mathbf{S}_{i}=\mathbf{H}_{i} \Lambda\left(k, \Delta h_{i}\right) \mathbf{H}_{i}^{-1}, \\
& \Delta h_{i}=h_{i}-h_{i+1} \quad(i=1,2, \ldots, n)
\end{aligned}
$$

The above matrices with subscript $i$ indicate the corresponding quantities with respect to the $i$ th-ply $(i=1,2, \ldots, n)$, respectively, $\Delta h_{i}$ is the $i$ th-ply thickness, and $h_{n+1}=0$ due to this interface located along the $x_{1}$ axis. The elements of matrix $\mathbf{B}$ can be extended in explicit form. From relations (24) and (27), the displacementtraction continuity along the interface (at $x_{2}=0$ ) between the $n$th layer and the half-space substrate leads to

$$
\begin{aligned}
& \left\{\begin{array}{l}
2 \mathrm{i} w\left(x_{1}-c t, 0\right) \\
\frac{2 \tau_{23}\left(x_{1}-c t, 0\right)}{\mathrm{i} k}
\end{array}\right\}^{(n)}=\left[\begin{array}{l}
\frac{1}{\mu_{n+1}} \\
1
\end{array}\right] G_{1}^{(n+1)} \exp [\mathrm{i} k(x-c t)], \\
& (-\infty<x<+\infty),
\end{aligned}
$$

where the superscript $(n+1)$ indicates the functions in half-space substrate. In the case of antiplane SAWs propagating in the above coating system, Equations (28) and (30) lead to

$$
\left\{\begin{array}{l}
2 \mathrm{i} w\left(x_{1}-c t, h_{1}\right) \\
0
\end{array}\right\}^{(1)}=\mathbf{B}\left[\begin{array}{l}
\frac{1}{\mu_{n+1}} \\
1
\end{array}\right] G_{1}^{(n+1)} \exp \left[\mathrm{i} k\left(x_{1}-c t\right)\right],
$$

which further yields the dispersion relation, i.e.

$$
B_{21}(k, p, \Delta h) / \mu_{n+1}+B_{22}(k, p, \Delta h)=0 .
$$

Equation (32) is the general dispersion relation of antiplane SAWs propagating in elastic half-space substrate coated with an anisotropic laminate, which may be extended into an explicit algebraic equation.

\section{Examples}

5.1. SAW dispersion relation for half-space coated with single anisotropic layer

In this case, there is only one anisotropic layer of thickness $h$, and Equation (32) reduces to

$$
\begin{gathered}
\tanh \left[k h \sqrt{C_{44}^{(1)}\left(C_{55}^{(1)}-\rho^{(1)} c^{2}\right)-C_{45}^{2}} / C_{44}^{(1)}\right] \\
-\frac{\sqrt{C_{44}^{(2)}\left(C_{55}^{(2)}-\rho^{(2)} c^{2}\right)-C_{45}^{(2)}} / C_{44}^{(2)}}{\sqrt{C_{44}^{(1)}\left(C_{55}^{(1)}-\rho^{(1)} c^{2}\right)-C_{45}^{(1)}} / C_{44}^{(1)}}=0,
\end{gathered}
$$

which is the dispersion relation of Love wave in anisotropic materials. It can be shown that there exist modes with phase velocities between the shear wave speeds of the two component materials, similar to the classic Love waves in isotropic materials [7-8]. Equation (33) shows that all Love waves are dispersive, and in the limiting case of isotropic materials, relation (33) covers the classic Love wave dispersion relation in the literature [7-8]. 


\subsection{SAW dispersion relation for half-space coated with two}

anisotropic layers

In this case, Equation (32) reduces to

$$
\frac{\sinh \left[k\left(h_{1} \mu_{1} / C_{44}^{(1)}-h_{2} \mu_{2} / C_{44}^{(2)}\right] \frac{\mu_{1}-\mu_{2}}{\mu_{1}+\mu_{2}}+\sinh \left[k\left(h_{1} \mu_{1} / C_{44}^{(1)}+h_{2} \mu_{2} / C_{44}^{(2)}\right]\right.\right.}{\cosh \left[k\left(h_{1} \mu_{1} / C_{44}^{(1)}-h_{2} \mu_{2} / C_{44}^{(2)}\right] \frac{\mu_{1}-\mu_{2}}{\mu_{1}+\mu_{2}}-\cosh \left[k\left(h_{1} \mu_{1} / C_{44}^{(1)}+h_{2} \mu_{2} / C_{44}^{(2)}\right]\right.\right.}+\frac{\mu_{3}}{\mu_{2}}=0,
$$

where $\mu_{i}(i=1,2,3)$ are defined in (13) for surface layers and the substrate, respectively, subscripts and superscripts 1, 2 and 3 denote respectively the physical quantities and thickness of the surface layers and the substrate. When material constants of surface layers keep the same values such that $C_{44}^{(1)}=C_{44}^{(2)}, C_{55}^{(1)}=C_{55}^{(2)}, C_{45}^{(1)}=C_{45}^{(2)}$, $\rho_{1}=\rho_{2}$, thus $\mu_{1}=\mu_{2}$ and relation (34) reduces to the anisotropic Love -wave dispersion relation (33) $\left(h_{1}+h_{2}=h\right)$.

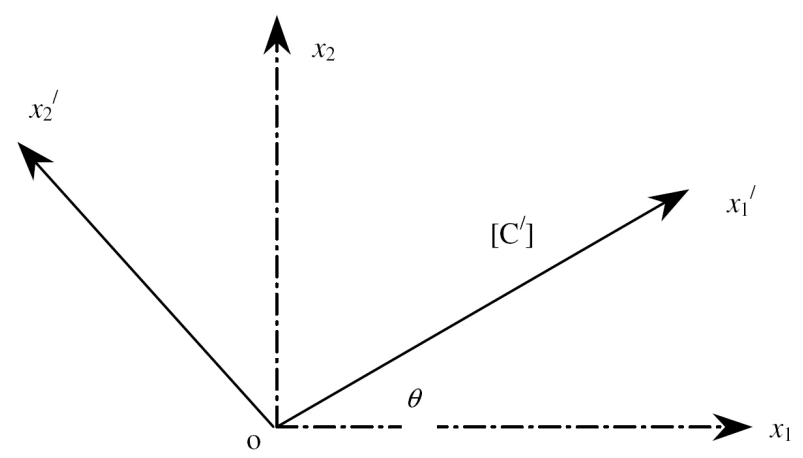

$[\mathrm{C}]$

Figure 2. Rotation of coordinate system along $x_{3}$-direction.

\subsection{SAW dispersion relation for half-space coated with an angle-ply composite laminate}

In Section 4 one can observes that the dispersion relations for antiplane SAWs propagating in elastic half-space coated with an anisotropic laminate can be obtained in explicit form by expanding relation (32). Here we consider the SAW dispersion relation of elastic half-space coated with an angle-ply laminate. The ply stiffness matrix for any lay-up angle may be determined by the matrix rotation between the local ply coordinate frame and the global coordinate frame of the laminate. Consider a rotation of the global coordinate frame $\left(x_{1}, x_{2}, x_{3}\right)$ along the $x_{3}$-axis to the local coordinate frame $\left(x_{1}^{\prime}, x_{2}^{\prime}, x_{3}^{\prime}\right)$, as shown in Figure 2.

The evoked stiffness elements $C^{\prime}{ }_{i j}$ for antiplane deformation in the new coordinate frame may be expressed in terms of $C_{i j}$ in the global coordinate frame as follows $[36,37]$ :

$$
\begin{aligned}
& C_{44}^{\prime}=\cos ^{2} \theta C_{44}-2 \sin \theta \cos \theta C_{45}+\cos ^{2} \theta C_{55}, \\
& C_{45}^{\prime}=\left(\cos ^{2} \theta-\sin ^{2} \theta\right) C_{45}+\sin \theta \cos \theta\left(C_{44}-C_{55}\right), \\
& C_{55}^{\prime}=\sin ^{2} \theta C_{55}+2 \sin \theta \cos \theta C_{45}+\sin ^{2} \theta C_{44},
\end{aligned}
$$

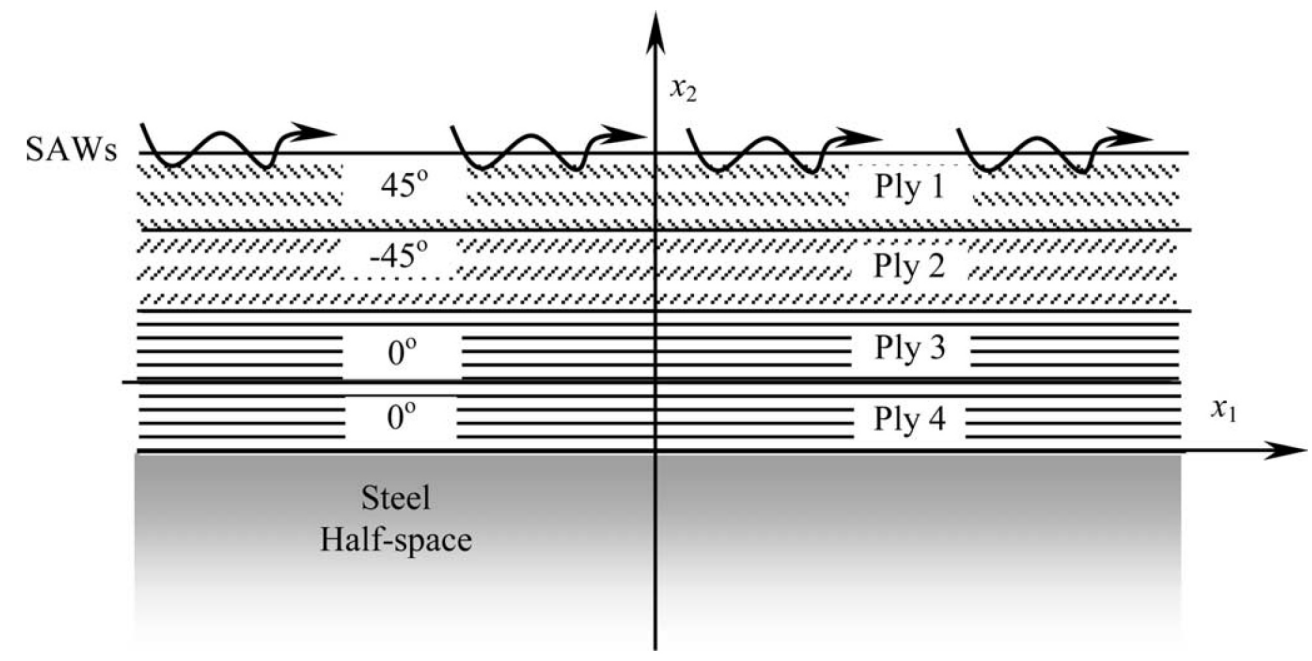

Figure 3. Antiplane SAWs propagating in elastic half-space coated with a $\left[ \pm 45^{\circ} / 0_{2}^{\circ}\right]$ composite laminate. 
Consider steel half-space covered with an angle-ply graphite-fiber/epoxy laminate made of Toray P7051S20Q-100 unidirectional prepregs. The mechanical properties of steel are $E=210 \mathrm{GPa}, v=0.28$, and the mass density $\rho=7800 \mathrm{~kg} / \mathrm{m}^{3}$. The mechanical properties of the unidirectional composite laminate in the local material coordinate system are $E_{1}=135 \mathrm{GPa}, E_{2}=E_{3}=8.5 \mathrm{GPa}, G_{12}=G_{\text {. }}$ ${ }_{3}=4.7 \mathrm{GPa}, G_{23}=3.03 \mathrm{GPa}, v_{12}=v_{13}=0.34, v_{23}=0.40$, the ply mass density is $\rho=2500 \mathrm{~kg} / \mathrm{m}^{3}$, and the ply thickness is $h=0.125 \mathrm{~mm}$. Therefore, the out-of-plane shear moduli in the material coordinate system, $\left(x_{1}=x_{2}\right)$-plane, are $C_{44}=3.03 \mathrm{GPa}, C_{45}=0, C_{55}=4.7 \mathrm{GPa}$. As an example, we consider the dispersion relation of the surface laminate with a $\left[ \pm 45^{\circ} / \mathrm{O}_{2}^{\circ}\right]$ lay-up as shown in Figure 3. The outof-plane shear moduli of plies with lay-up angle $\pm 45^{\circ}$ are determined from (35).

The dispersion relation can be determined straightforwardly by expanding Equation (32) such that

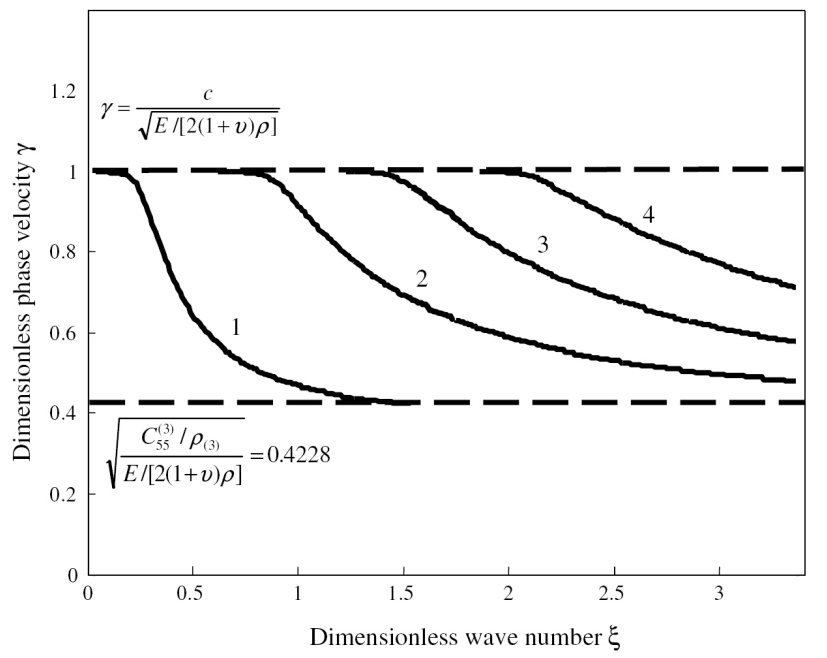

Figure 4. Dispersion relation of antiplane SAWs in steel halfspace coated with a $\left[ \pm 45^{\circ} / 0_{2}^{\circ}\right]$ composite laminate.

$$
\frac{\sinh \left[2 k h\left(\mu_{1} / C_{44}^{(1)}-\mu_{3} / C_{44}^{(3)}\right] \frac{\mu_{3}-\mu_{1}}{\mu_{3}+\mu_{1}}+\sinh \left[2 k h\left(\mu_{1} / C_{44}^{(1)}+\mu_{3} / C_{44}^{(3)}\right]\right.\right.}{\cosh \left[2 k h\left(u_{1} / C_{44}^{(1)}-u_{3} / C_{44}^{(3)}\right] \frac{\mu_{3}-\mu_{1}}{\mu_{3}+\mu_{1}}+\cosh \left[2 k h\left(u_{1} / C_{44}^{(1)}+u_{3} / C_{44}^{(3)}\right]\right.\right.}-\frac{\mu}{\mu_{3}}=0,
$$

where $\mu=\left(E /[2(1+v)]\left\{E /[2(1+v)]-\rho c^{2}\right\}\right)^{1 / 2}$, and $\rho, E$, and $v$ are, respectively, the mass density, Young's modulus, and Poisson's ratio of the steel substrate. Relation (36) is similar to (34) due to the $\pm 45^{\circ}$ plies bearing the similar out-of-plane properties. When the phase velocity $c$ keep $\mu$ a pure imaginary, the corresponding dispersive wave does not have energy leakage into the substrate.

In the present case $C_{44}^{(1)}=C_{55}^{(1)}=3.865 \mathrm{GPa}, C_{45}^{(1)}=$ $-0.865 \mathrm{GPa}, C_{44}^{(3)}=3.03 \mathrm{GPa}, C_{55}^{(3)}=4.7 \mathrm{GPa}$, and $C_{45}^{(3)}=$ $0.0 \mathrm{GPa}$. By introducing a dimensionless wave number and a dimensionless phase velocity such that

$$
\xi=2 k h, \quad \gamma=c / \sqrt{E /[2(1+v) \rho]},
$$

the subsonic non-leakage wave dispersion curves of the first four modes are plotted in Figure 4. The numerical procedure for finding the roots of wave number at given phase speed was performed using the simple searching method. This method was proved very robust for the present problem.

From Figure 4, one can find that for each subsonic wave mode, the wave phase speed tends to the shear wave speed of the half-space substrate (stiffer) when the wave number tends to zero, while it tends to the shear wave speed of $0^{\circ}$-ply close to the substrate when the wave number is very large. The $0^{\circ}$-ply has the highest shear wave speed in the surface laminate. Consequently, from the dispersion relation (36), one can also observe that non-leakage SAWs only exist under condition that the wave speed of the substrate is higher than that of any surface ply. This is similar to the existence condition of classic Love waves. The above two wave speeds form the upper and lower limits of the subsonic non-leakage SAWs in multilayered anisotropic coating systems.

\section{Concluding remarks}

By means of Stroh's formalism of anisotropic elasticity, explicit dispersion relations of antiplane SAWs propagating in elastic half-space coated with an anisotropic laminate have been determined. The existence condition of subsonic non-leakage SAWs has been given. It can be verified that this condition also holds for inplanar SAW cases. It should be mentioned that for inplanar cases the eigenvalue problems have to be involved for determining the corresponding Stroh's functions. Since the given results in this work are exact ones, they are applicable for examining the entire subsonic frequency range of anisotropic coating systems. The exact dispersion relations can be used as the theoretical basis for the evaluation of other approximate methods and NDE based on surface/ guided wave methods. Furthermore, this method can be easily furthered for the study of steady waves propagating in layered surface coatings and composite laminates with damages such as delamination.

\section{Acknowledgments}

Partial support of this work by the U.S. Army Research Office and the U.S. Air Force Office of Scientific Research is gratefully acknowledged. The authors would like to thank Professors R. Feng, J. A. Turner, and J. S. Yang at the Department of Engineering Mechanics, University of Nebraska-Lincoln, for their helpful discussions and suggestions. The authors would like to thank the four anonymous reviewers from Composites Science and Technology for their enlightening comments and detailed suggestions for the improvement of the present manuscript. 


\section{References}

1. A. Briggs, Acoustic microscopy, Clarendon Press, Oxford (England) (1992).

2. L. W. J. Schmerr, Fundamentals of ultrasonic nondestructive evaluation: a modeling approach, Plenum Press, New York (1998).

3. D.C. Hurley, V. K. Tewary, and A. J. Richards, Surface acoustic wave methods to determine the anisotropic elastic properties of thin films, Meas Sci Technol 12 (2001), pp. 1486-1494.

4. D. C. Hurley, K. Shen, N. M. Jennett, and J. A. Turner, Atomic force acoustic microscopy methods to determine thin-film elastic properties, J Appl Phys 94 (2003), pp. 2347-2354.

5. A. G. Every, Measurement of the near-surface elastic properties of solids and thin supported films, Meas Sci Technol 13 (2002), pp. R21-R39.

6. A. E. H. Love, Some problems in geodynamics, Cambridge Press, Cambridge (England) (1911).

7. J. D. Achenbach, Wave propagation in elastic solids, Elsevier, New York (1973).

8. K. F. Graff, Wave motion in elastic solids, Dover Publications, Inc., New York (1991).

9. I. A. Viktorov, Rayleigh and lamb waves, Plenum Press, New York (1967).

10. B. A. Auld, Acoustic fields and waves in solids, vol. 2, Krieger Publishing Company, Florida (1990).

11. A. L. Shuvalov and A. G. Every, Some properties of surface acoustic waves in anisotropic-coated solids, studied by the impedance method, Wave Motion 36 (2002), pp. 257-273.

12. P. Chadwick and G. D. Smith, Foundations of the theory of surface waves in anisotropic elastic materials, Adv Appl Mech 17 (1977), pp. 303-376.

13. D. M. Barnett and J. Lothe, Free surface (Rayleigh) waves in anisotropic elastic half-spaces: the surface impedance methods, Proc Roy Soc (London) 402A (1985), pp. 135-152.

14. T. C. T. Ting, Anisotropic elasticity: theory and applications, Oxford University Press, Oxford (England) (1996).

15. L. M. Brekhovskikh, Waves in layered media, Academic Press, New York (1960).

16. L. M. Brekhovskikh and O. A. Godin, Acoustics of layered media I: Plane and quasi-plane waves, Springer-Verlag, Heidelberg (1990).

17. G. W. Farnell, Properties of elastic surface waves, Phys Acoustics 6 (1970), pp. 109-166.

18. G. W. Farnell and E. L. Adler, Elastic wave propagation in thin layers. In: W. P. Farnell Mason and R. W. Adler Thurston, Editors, Physical acoustics, Academic Press, New York (1972), pp. 35-127.
19. H. Murakami, A mixture theory for wave propagation in angle-ply laminates, Part I: Theory, ASME J Appl Mech 52 (1985), pp. 333-337.

20. H. Murakami and A. Akiyama, A mixture theory for wave propagation in angle-ply laminates, Part II: Applications, ASME J Appl Mech 52 (1985), pp. 338-344.

21. G. R. Liu, J. Tani, K. Watanabe, and T. Ohyoshi, Lamb wave propagation in anisotropic laminates, ASME J Appl Mech 57 (1990), pp. 923-929.

22. G. R. Liu, J. Tani, K. Watanabe, and T. Ohyoshi, Harmonic wave propagation in anisotropic laminated strips, J Sound Vib 139 (1990), pp. 313-324.

23. G. R. Liu and Z. C. Xi, Elastic waves in anisotropic laminates, CRC Press, New York (2002).

24. E. L. Adler, J. K. Slaboszewicz, G. W. Farnell, and C. K. Jen, PC software for SAW propagation in anisotropic multilayers, IEEE Trans Ultra Ferro Freq Cont 37 (1990), pp. 215-223.

25. E. L. Adler, Matrix methods applied to acoustic waves in multilayers, IEEE Trans Ultra Ferro Freq Cont 37 (1990), pp. 485-490.

26. P. M. Smith, Dyadic Green's functions for multi-layer SAW substrates, IEEE Trans Ultra Ferro Freq Cont 48 (2001), pp. 171-179.

27. E. L. Tan, A robust formulation of SAW Green's functions for arbitrarily thick multilayers at high frequencies, IEEE Trans Ultra Ferro Freq Cont 49 (2002), pp. 929-936.

28. C. T. Sun, J. Luo, and R. W. McCoy, Analysis of wave propagation in thick-section composite laminates using effective moduli, Composites B-Eng 27 (1996), pp. 613-621.

29. G. Caviglia and A. Morro, Wave propagation in multilayered anisotropic solids, Int J Eng Sci 38 (2000), pp. 847-863.

30. G. Caviglia and A. Morro, Reflection and transmission in pre-stressed stratified multilayers, Acta Mech 153 (2002), pp. 113-126.

31. A. H. Nayfeh, Wave propagation in layered anisotropic media, North-Holland, New York (1995).

32. A. N. Stroh, Dislocations and cracks in anisotropic elasticity, Philos Mag 7 (1958), pp. 625-646.

33. A. N. Stroh, Steady-state problems in anisotropic elasticity, J Math Phys 41 (1962), pp. 77-103.

34. S. G. Lekhnitskii, Theory of elasticity of an anisotropic body, Holden-Day, San Francisco (1963).

35. K. C. Wu, Anti-plane shear interface cracks in anisotropic bimaterials, ASME J Appl Mech 58 (1991), pp. 399-403.

36. R. M. Christensen, Mechanics of composite materials, Krieger Publishing Company, Florida (1979).

37. M. J. Jones, Mechanics of composite materials (2nd ed.), Taylor \& Francis, Philadelphia (1998). 